\title{
Effects of fixed cutoff filtering on dark- and light-adapted ERG components and the application of variable cutoff filter
}

\author{
Min Gao • Mirella Telles Salgueiro Barboni · Dora Fix Ventura • \\ Balázs Vince Nagy (10
}

Received: 5 August 2020/ Accepted: 10 September 2021/Published online: 24 September 2021

(C) The Author(s) 2021

\begin{abstract}
Purpose Human oscillatory potentials (OPs) are derived from dark-adapted (DA) electroretinograms (ERGs) with fixed frequency cutoff filters while lightadapted (LA) OPs are generally not isolated from ERGs. Our purpose was to analyze the effect of cutoff frequencies on DA and LA ERG components using a series of fixed and variable filters.

Methods DA and LA ERGs were recorded from 10 healthy eyes of 10 subjects (mean age $=20.5 \pm 6.7$ years) following ISCEV standards. Each signal was filtered in the Fourier domain to acquire slow (a- and b-waves; below cutoff frequency) and fast (OPs; above cutoff frequency) components. Fixed cutoff frequencies ranged from 60 to $105 \mathrm{~Hz}$ and a variable cutoff frequency was calculated. Results were analyzed with statistical tests and specific models.

Results DA ERG components were slightly influenced by the filter cutoff frequency. In contrast, fixed
\end{abstract}

M. Gao · B. V. Nagy $(\bowtie)$

Department of Mechatronics, Optics and Mechanical

Engineering Informatics, Budapest University of

Technology and Economics, Budapest, Hungary

e-mail: nagyb@mogi.bme.hu

\section{T. S. Barboni}

Department of Ophthalmology, Semmelweis University, Budapest, Hungary

M. T. S. Barboni · D. F. Ventura

Department of Experimental Psychology, University of Sao Paulo, Sao Paulo, Brazil and variable filters significantly changed LA components: the lower the cutoff frequency the smaller the b-wave and OP3 and the higher the OP2/OP4 amplitudes. Analyzing the filter frequency limits a transition range between $68.9 \mathrm{~Hz}$ and $83.9 \mathrm{~Hz}$ was observed where amplitudes vary.

Conclusions The present report shows that DA OPs may be isolated from ERGs using filtering procedures with high-pass cutoff frequency at about $75 \mathrm{~Hz}$ as recommended by ISCEV. On the other hand, the spectral distribution of low-frequency and high-frequency LA ERG components may overlap. Accordingly, filtering the signal using different cutoff frequencies is not necessarily separating b-wave and OPs.

Keywords Retina $\cdot$ Electroretinogram $\cdot$ Oscillatory potentials · ISCEV standard · Spectral analysis · Cutoff frequency $\cdot$ Variable filter

\section{Introduction}

The electroretinogram (ERG) is a mass retinal bioelectric potential elicited by flashes of light with a combination of slow and fast components. Digital filters applied to ERG signals have been in use for a long time in an attempt to identify signal components, such as oscillatory potentials (OPs), that are hardly 
detected within the full signal spectrum usually recorded in the clinical routine [1-7]. Although, the cutoff frequencies to extract OPs from the original digitized dark-adapted (DA) ERG signals having a large range of frequencies up to $300 \mathrm{~Hz}$ is standardized by ISCEV, the influence of higher cutoff frequencies on OPs is still debated [8-10]. Moreover, light-adapted (LA) OPs are not necessarily extracted from the original digitized ERG signals during clinical examinations [11]. The fast OP components are usually observed when the slow components are reduced or eliminated by filtering out low-frequencies, for instance below $75 \mathrm{~Hz}$, as recommended by the ISCEV, for the time domain analysis of DA OPs [11].

On the other hand, high-frequency components (OPs) are usually not filtered out from the original digitized ERG signals when the low-frequency components (a-wave and b-wave) are analyzed in the time domain. Although for most clinical cases this might not be relevant, OPs overlapping the slow components may influence the selection of a-wave and b-wave peaks in retinal conditions.

The purpose of this study was to evaluate the effects of different cutoff frequencies to DA and LA ERG components considering low and high frequency signal components. While OP changes associated with the cutoff frequency have been well described, the influence of the high-frequency components to the human a-wave and the b-wave is less known. We selected a range of frequencies (60 to $105 \mathrm{~Hz}$ ) based on a previous report showing specific bands of temporal frequencies in the power spectrum of LA ERG signals [12]. In addition, a variable filter, as used in murine ERGs [13] has been applied.

\section{Methods}

\section{Data collection}

The experiment was performed in accordance with the declaration of Helsinki and approved by the institutional ethics committee. Participants were 10 healthy volunteers (mean age $=20.5 \pm 6.7$ years old; four males and six females) signing a consent to participate in the study after the explanation of the nature and possible consequences of the study. ERGs were recorded from one randomly selected eye using the RETiport system (Roland Consult, Brandenburg, Germany) equipped with Q450 SC Ganzfeld stimulator after i) eye dilation (tropicamide 1\%), ii) electrodes placement: active DTL fiber electrode attached from the outer to the inner canthus, and ground / reference skin electrodes on the external canthi and forehead, respectively, and iii) a $20 \mathrm{~min}$ period of dark-adaptation. ERGs to dark-adapted (DA) and light-adapted (LA) $3.0 \mathrm{~cd} . \mathrm{s} / \mathrm{m}^{2}$ flash intensity $\left(30 \mathrm{~cd} / \mathrm{m}^{2}\right.$ background luminance delivered for $10 \mathrm{~min}$ prior to LA recordings) were recorded according to ISCEV standards [11]. ERG signals with 512 points in $256 \mathrm{~ms}$, including a $16 \mathrm{~ms}$ pre-stimulus, were obtained.

Signal analysis

Offline signal analysis was performed with selfwritten Matlab ${ }^{\circledR}$ (Mathworks, Natick, MA, USA) programs. Simple bandpass filters using Fast Fourier Transform (FFT) and Inverse Fast Fourier Transform (IFFT) were used. The spectral distribution with a bandwidth from 1 to $300 \mathrm{~Hz}$ in increments of $3.9 \mathrm{~Hz}$, selecting only the frequency values to obtain approximately $5 \mathrm{~Hz}$ intervals from 60 to $105 \mathrm{~Hz}$, were defined as cutoff frequencies for the fixed filter to separate the full range (1 to $300 \mathrm{~Hz}$ ) into two frequency bands. Then the two new signals in the time domain: the low-frequency signal with slow components (a- and b-wave), and the high-frequency signal with fast components (OPs), were obtained with the IFFT. In addition, the spectral distribution of each individual signal was used to find the frequency with the first minimum amplitude between 60 and $90 \mathrm{~Hz}$. This frequency was selected as the cutoff frequency for the application of the variable filter [13]. The original signals as well as the filtered signals were analyzed in the time-domain. The negative amplitudes (a-wave) were measured from the baseline (average amplitude of the first $5 \mathrm{~ms}$ recorded before the flash onset) to the trough while the amplitudes of the positive components (b-wave and positive OP peaks) were measured from the trough of the preceding negative peak. Peak times were measured from the flash onset to the peak amplitude of each component. Figure 1 shows average signals with components analyzed in the time domain (A: DA response and B: LA response) and the spectral distribution in the frequency domain obtained with the FFT (C: DA response and D: LA response). 
Fig. 1 Averaged darkadapted (a) and lightadapted (b) original ERG signals and OPs derived from the original ERG signals using a series of fixed high-pass cutoff filters from 60 to $105 \mathrm{~Hz}$ every $5 \mathrm{~Hz}$. The name of the slow components (a-wave and b-wave) and the fast components (OP1 to OP5) analyzed are given. Averaged spectral power distributions are shown for the dark-adapted (c) and the light-adapted (D) ERG
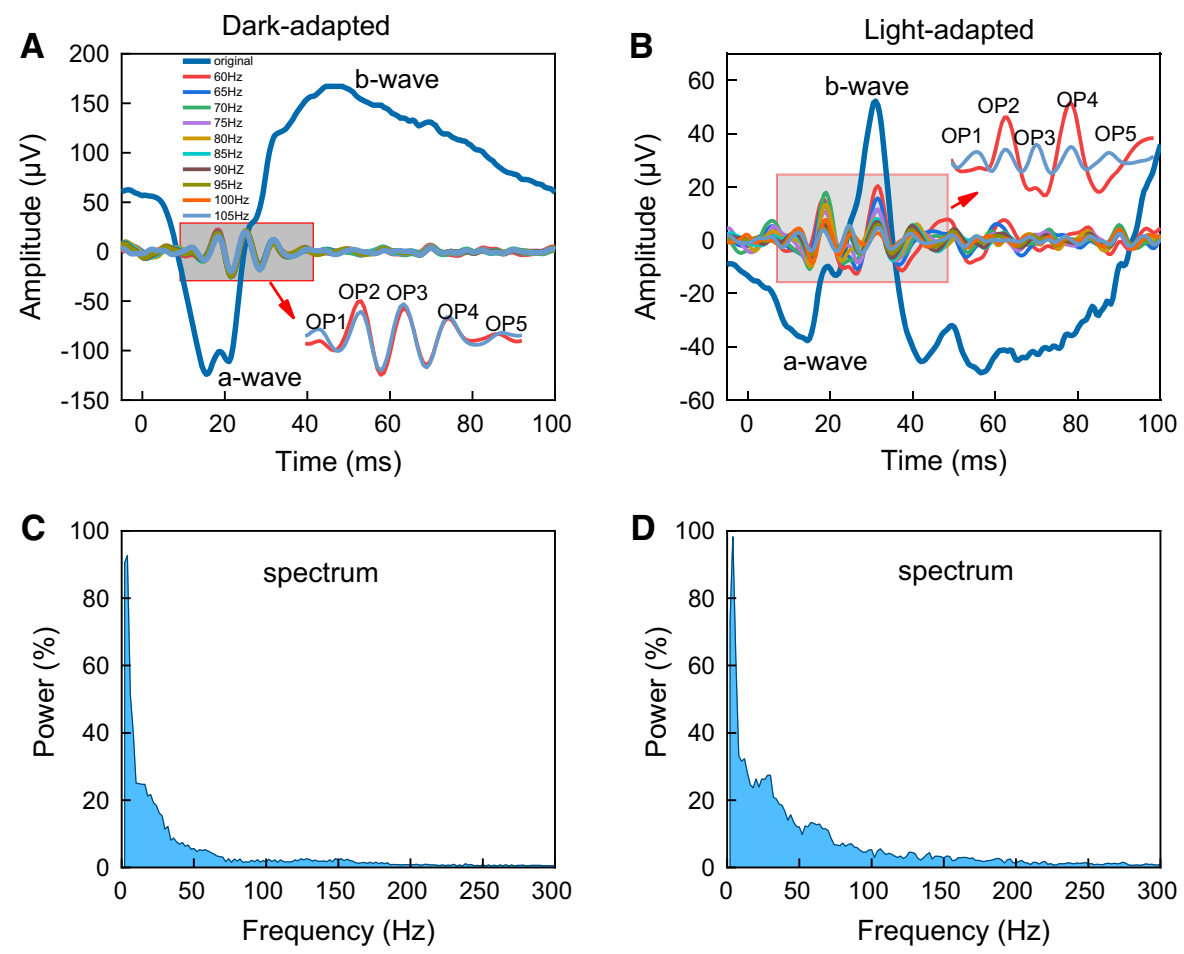

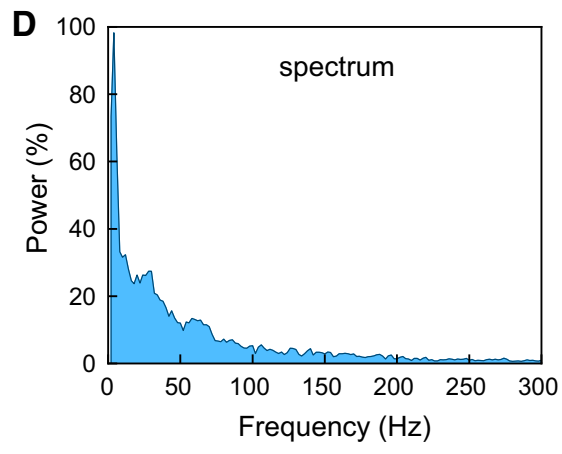

Statistical analysis

Data are expressed as means \pm standard error of the mean. The effects of cutoff frequency filtering on ERG components were evaluated using Wilcoxon tests and paired t-test comparisons with Bonferroni post-hoc analyses (SPSS, Statistical Package for the Social Sciences, Hong Kong, China); multiple comparison corrected $\mathrm{p}$ values $<0.001$ were considered statistically significant. In addition, Hill functions were fitted on measurement data, where applicable, to calculate the $50 \%$ thresholds and corresponding frequencies.

\section{Results}

Time-to-frequency and frequency-to-time domain analysis using fixed filters

Mean dark-adapted (DA; Fig. 1a) and light-adapted (LA; Fig. 1b) original ERG signals and OPs derived from the original ERG signals are shown in Fig. 1. Table 1 shows the mean ( \pm standard error) of each component obtained with the original signals measured in the time domain. OPs were derived from the original ERG signals using a series of 10 fixed highpass cutoff filters from 60 to $105 \mathrm{~Hz}$. Individual timedomain amplitudes and peak times were calculated for each filter applied. Five oscillatory potentials were identified in both DA (Fig. 1a) and LA (Fig. 1b) signals. Peak time of the DA OP1 and OP2 coincided with a-wave peak time while DA OP3, OP4, and OP5 were superimposed to the rising slope of the signal which originates the b-wave. OP3 was usually the highest in amplitude (Fig. 1a, colored traces superimposed onto the original signal). LA OP1 was also superimposed onto the a-wave while OP2, OP3, and OP4 were superimposed onto the ascending limb of the b-wave. OP5 is after the b-wave. One may observe that LA OP amplitudes were largely and differently affected by the filtering condition (Fig. 1b), contrary to what was seen in DA OP responses (Fig. 1a). LA OP2 and OP4 amplitudes were about $49 \%$ and 52\% lower at $105 \mathrm{~Hz}$ when compared to the amplitudes obtained at the $60 \mathrm{~Hz}$ cutoff frequency while LA OP3 amplitudes increased 54\%, in average, at $105 \mathrm{~Hz}$ compared to the average at the $60 \mathrm{~Hz}$ cutoff frequency. LA OP3 amplitudes were larger than LA OP2 and OP4 amplitudes at $105 \mathrm{~Hz}$ cutoff frequency (Fig. 1b). 
Table 1 DA and LA amplitudes and peak times (mean \pm standard error) of the a-wave and the b-wave obtained from the original signals and after applying the variable filter

\begin{tabular}{|c|c|c|c|c|c|}
\hline & & \multicolumn{2}{|l|}{ Original } & \multicolumn{2}{|l|}{ Variable filter } \\
\hline & & Amplitude $(\mu \mathrm{V})$ & Peak time (ms) & Amplitude $(\mu \mathrm{V})$ & Peak time (ms) \\
\hline \multirow[t]{2}{*}{ Dark-adapted } & a-wave & $197.6 \pm 18.1$ & $16.5 \pm 0.7$ & $196.2 \pm 21.1$ & $16.7 \pm 0.4$ \\
\hline & b-wave & $305.9 \pm 36.1$ & $45.7 \pm 2.6$ & $298.7 \pm 36.7$ & $40.0 \pm 1.8$ \\
\hline \multirow[t]{2}{*}{ Light-adapted } & a-wave & $42.4 \pm 8$ & $13.9 \pm 0.6$ & $38.3 \pm 6.1$ & $11.7 \pm 0.6$ \\
\hline & b-wave & $114.2 \pm 11.1$ & $30.5 \pm 0.4$ & $98.4 \pm 10.1$ & $30.5 \pm 0.3$ \\
\hline
\end{tabular}

Group averages of the spectral power distribution are shown in Fig. 1c (DA) and Fig. 1d (LA). DA and LA spectra differ in spectral power distribution especially between 60 and $105 \mathrm{~Hz}$ where the averaged LA power is more than twice as large (207\%) as the average DA power.

Time-to-frequency and frequency-to-time domain analysis using the variable filter

In addition to the 10 fixed cutoff filters, a variable filter method has been applied to each individual signal. As previously described in the methods [13], the filter aimed to select the cutoff frequency as the first minimum amplitude of the spectrum calculated from each individual ERG signal. Figure 2 shows the analysis of one representative subject for the DA (Fig. 2a) and LA (Fig. 2b) responses. Similarly, to the analysis with the fixed cutoff filters, slow and fast components were derived from the original signals providing a-wave, b-wave, and OPs to be analyzed in the time domain. An individual spectral distribution is shown (Fig. 2) with red arrows indicating the minimum frequency between 60 and $90 \mathrm{~Hz}$ where the transition between slow and fast component separation generally occurs. The minimum FFT amplitude ranged from 72 to $85 \mathrm{~Hz}$ in both DA (mean $=78.2 \pm$ $1.7 \mathrm{~Hz})$ and $\mathrm{LA}($ mean $=76.8 \pm 1.4 \mathrm{~Hz})$ conditions. Therefore, each individual signal showed a slightly different minimum frequency. Mean DA and LA slow component (a- and b-wave) amplitudes and peak time obtained from the variable filter are shown in Table 1 and were not statistically different from those of the values obtained with the original signal $(\mathrm{p} \geq 0.02)$.

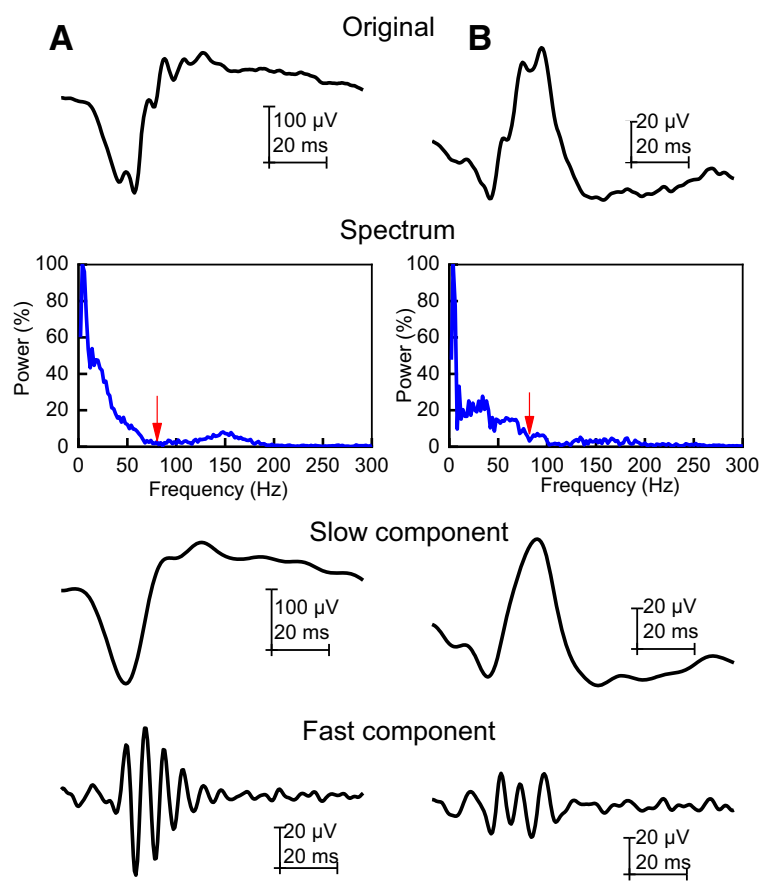

Fig. 2 Variable filter applied to one representative subject for the DA (a) and LA (b) responses. The original ERG signals in the time domain are shown in the top row. Using the spectral power distribution (second row) the first minimum amplitude between 60 and $90 \mathrm{~Hz}$ was selected (indicated with the red arrow: $84 \mathrm{~Hz}$ and $82 \mathrm{~Hz}$, respectively) as the cutoff frequency. The isolated low-frequency signal and high-frequency signal obtained from the original ERG signal using the frequencies below (slow component) and above (fast component) the cutoff frequency is shown in the third and fourth rows

Effects of the cutoff frequency on the slow (a-wave and b-wave) and fast (OPs) components of the DA ERG

DA slow and fast components varied slightly in waveform among the 10 fixed filters. Figures 3 and 4 show mean ( \pm standard error) amplitudes (upper 
Fig. 3 Dark-adapted slow components (a-wave and b-wave) response as a function of cutoff frequencies. Mean $( \pm$ one standard error) amplitudes (a, b) and peak times (c, d) of the slow components (a-wave $=\mathrm{A}$ and b-wave = B) for each fixed cutoff frequency (connected by black symbols) and average ( \pm standard error) variable filter (isolated blue symbol) are shown. Mean ( \pm standard error) values obtained with the original signals are shown with horizontal gray lines and dotted lines

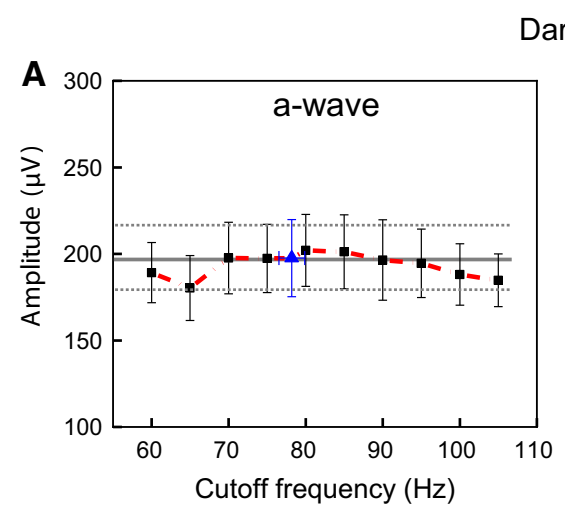

Dark-adapted
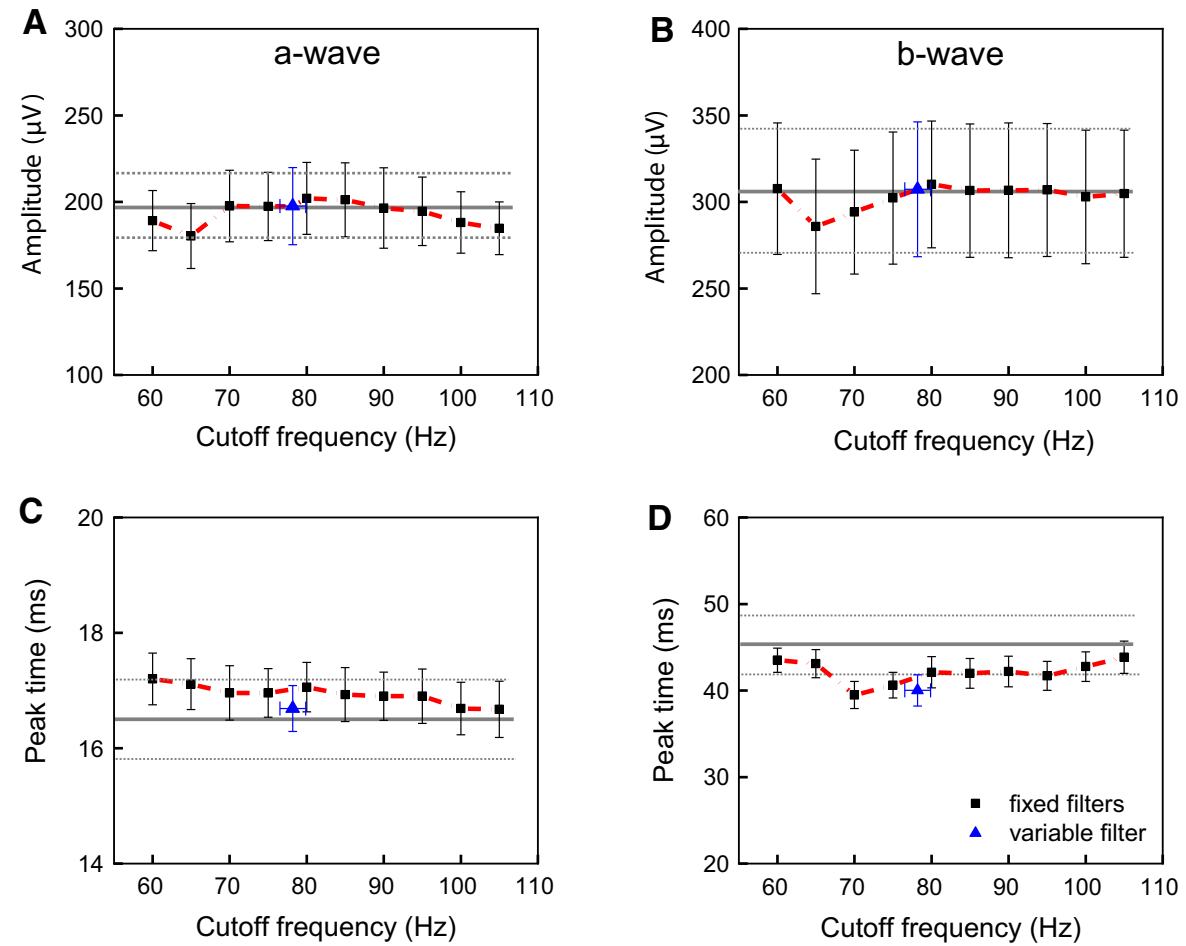

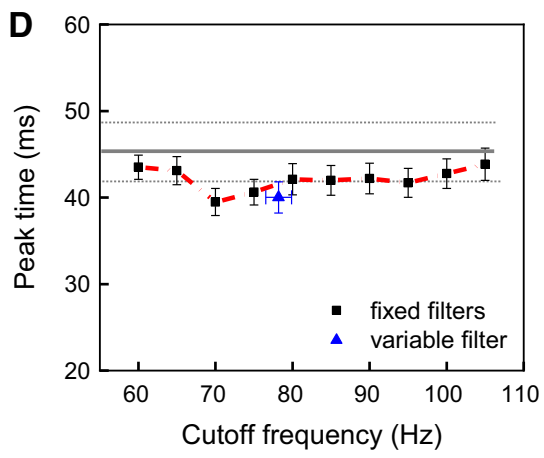

plots) and peak times (lower plots) of the slow (a-wave and b-wave) and fast (OP2, OP3, OP4) components at each cutoff frequency set for the fixed filters (connected black symbols with short dash line), and the variable filter (isolated blue symbols). The mean ( \pm standard error) values of the original a-wave and b-wave amplitudes and peak times are also displayed (horizontal gray lines and dotted lines).

The amplitudes of DA a- wave (Fig. 3a) and b-wave (Fig. 3b) did not significantly differ among the 10 filters applied and compared to the results obtained with the original signals $(p>0.02)$. In addition, the variable filter $($ mean $=78.2 \pm 5.3 \mathrm{~Hz})$ resulted in similar amplitude values (a-wave: $p>0.26$; b-wave: $p>0.19)$ compared to the fixed filters. Peak times (Fig. $3 \mathrm{c}$ and d) were statistically similar among the 10 fixed cutoff filters (a-wave $p>0.14$ and b-wave $p>0.98$ ), although they were lower compared to the original signal for the b-wave, as shown in Fig. 3d.

Figures $4 \mathrm{~A}$ to $4 \mathrm{C}$ show that DA OP2, OP3, OP4 amplitudes, respectively, obtained with ten fixed filters (OP2: $p>0.03$; OP3: $p>0.09$; OP4: $p>0.06)$ and the variable filter (OP2: $p>0.02$;
OP3: $p>0.16$; OP4: $p>0.03$ ), did not significantly change, considering correction for multiple comparisons (to reach statistical significance p-value should be $<0.001)$. Peak times were relatively constant for each fast component among fixed and variable filters (OP2: $p>0.07$; OP3: $p>0.04$; OP4: $p>0.11$ ) as shown in Figs. $4 \mathrm{~d}-\mathrm{f}$.

Effects of the filters on the slow (a-wave and bwave) and fast (OPs) components of the LA ERG

Contrary to the effects on DA ERG, LA slow and fast components significantly changed with the filters. Figures 5 and 6 show the mean ( \pm standard error) amplitudes (upper plots) and peak times (lower plots) of the LA slow (a-wave and b-wave) and the fast (OPs) components for the fixed filters with different cutoff frequencies (connected black symbols), and for the variable filter (isolated blue symbols). The mean ( \pm standard error) values of the original a-wave and b-wave amplitudes and peak times are also displayed (horizontal gray lines and dotted lines).

Hill equation was applied to analyze LA amplitudes of all filtered components. The fitting curves show "S- 
Dark-adapted
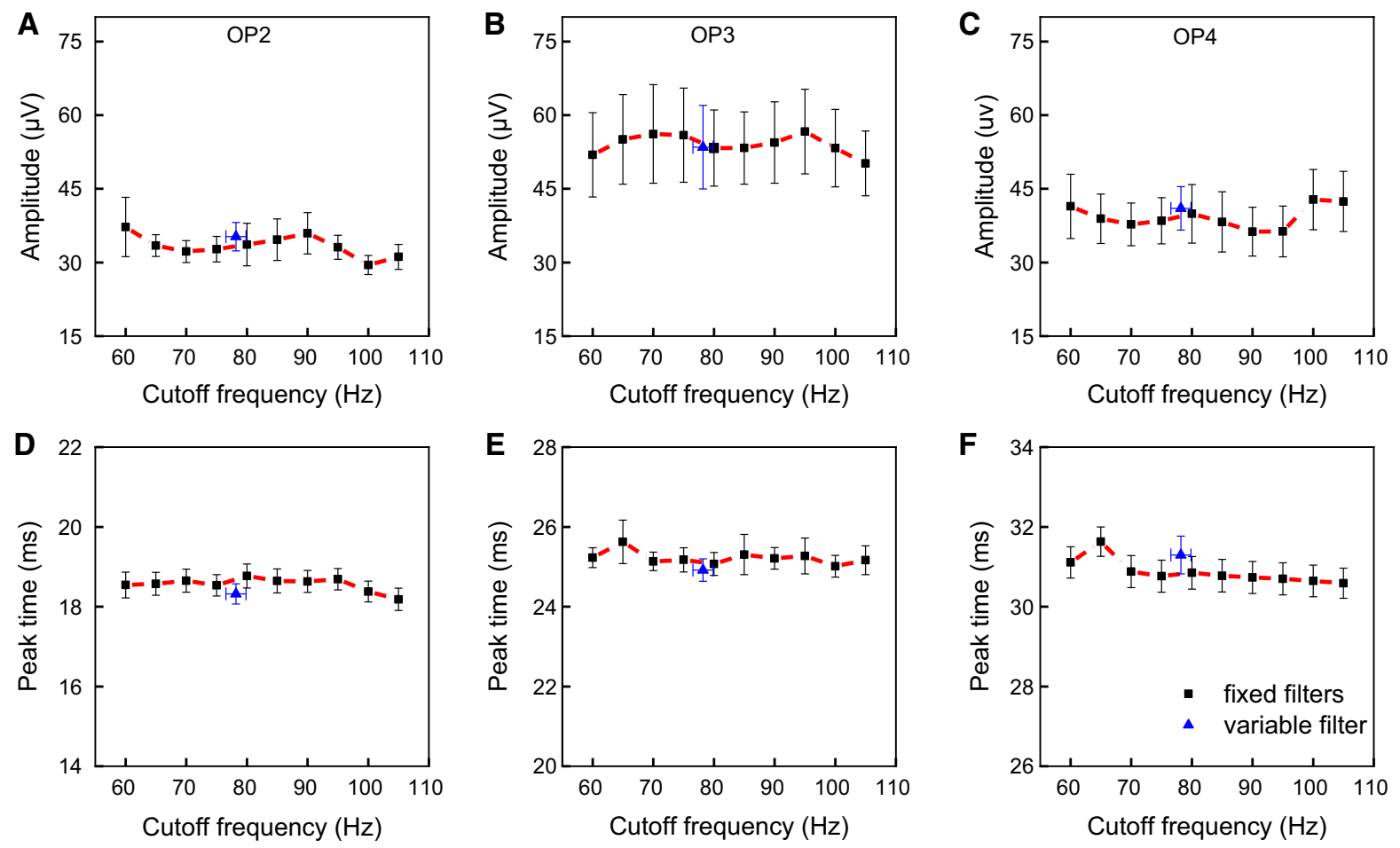

Fig. 4 Dark-adapted fast components responses as a function of cutoff frequencies. Mean ( \pm one standard error) amplitudes (upper plots) and peak times (lower plots) of the fast $(\mathbf{a}=\mathrm{OP} 2$,

like" shapes, which means there's a linear range corresponding to the middle section (transition range) and two relatively constants sections at low and high frequencies. LA a-wave amplitudes varied among the fixed filters with the 50\% threshold at $76.23( \pm 8.90)$ $\mathrm{Hz}$, but not significantly ( $p=0.16$, comparing the lowest amplitude at $65 \mathrm{~Hz}$ with the highest amplitude at $105 \mathrm{~Hz}$ ), as shown in Fig. 5a. In addition, a-wave amplitudes obtained with the fixed filters did not differ statistically from the original a-waves $(p>0.02)$, considering correction for multiple comparisons (to reach statistical significance p-value should be $<$ 0.001). The amplitude of $b$-wave is shown in Fig. $5 b$. The results transited from a relatively constant low sections to a high section with the 50\% threshold at $79.4( \pm 4.5) \mathrm{Hz}$, in average. The b-wave amplitudes obtained with cutoff frequencies higher than $80 \mathrm{~Hz}$ were not significantly different from the original signal's b-wave $(p>0.30)$. Moreover, amplitudes obtained with the fixed filters and the variable filter $(76.8 \pm 1.4 \mathrm{~Hz})$ did not differ significantly (a-wave:
$\mathbf{b}=\mathrm{OP} 3$ and $\mathbf{c}=\mathrm{OP} 4)$ components for each cutoff frequency (connected black symbols) and average ( \pm standard error) variable filter (isolated blue symbols) are shown

$p>0.07$; b-wave: $p>0.02$ ). On the other hand, amplitudes obtained with low and high cutoff frequencies (60 Hz vs. 80-105 Hz $p<0.0007 ; 65 \mathrm{~Hz}$ vs. $95-105 \mathrm{~Hz} \quad p<0.0004 ; 70 \mathrm{~Hz}$ vs. $100-105 \mathrm{~Hz}$ $p<0.0001 ; 80 \mathrm{~Hz}$ vs. $95-105 \mathrm{~Hz} p<0.0006)$ differed significantly from each other, indicating significant effect of the filter frequency on LA b-wave amplitudes. Peak times of the slow components (Figs. 5c and d) varied among the 10 fixed cutoff filters (a-wave: $95 \mathrm{~Hz}$ vs. $100-105 \mathrm{~Hz} \mathrm{p}<0.0004$, and b-wave: $65 \mathrm{~Hz}$ vs. $80-95 \mathrm{~Hz} p<0.0006$ ) indicating significant effect of the filter frequency on LA slow components. Moreover, the peak times of both a-wave and b-wave obtained with the fixed filters and the variable filter $(76.8 \pm 1.4 \mathrm{~Hz})$ did not differ significantly (a-wave: $p>0.09$; b-wave: $p>0.001$ 0.99) Statistical values showed comparable a-wave peak times, although in Fig. $5 \mathrm{c}$ the mean value obtained with the variable filter was below the low standard error value of the mean a-wave peak time obtained with original signals. However, we assume 
Fig. 5 Light-adapted (LA) slow components (a-wave and b-wave) as a function of cutoff frequencies. Mean ( \pm standard error) amplitudes (a and b) and peak times (c and d) for each cutoff frequency (connected black symbols) and the average ( \pm standard error) variable filter (isolated blue symbols) were shown. Mean ( \pm standard error) values obtained with the original signals are shown with horizontal gray lines and dotted lines. Hill function was applied to the a- and b-wave amplitudes (a and b) showing that amplitudes increase as a function of cutoff frequency achieving a level at about $85 \mathrm{~Hz}$ with the $50 \%$ threshold at $(76.2 \pm 8.9) \mathrm{Hz}$ and 79.4 $( \pm 4.5) \mathrm{Hz}$, respectively
Light-adapted
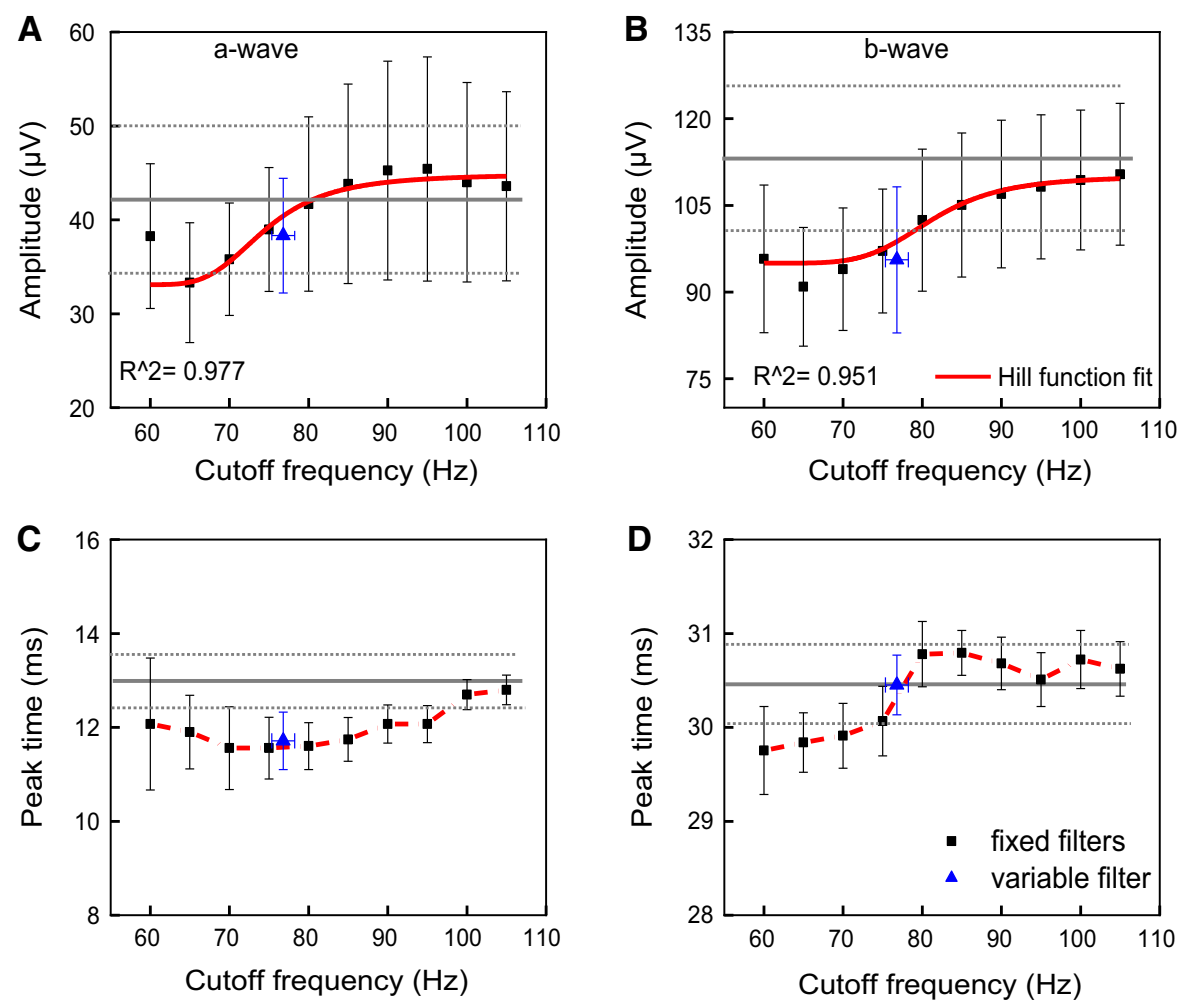

that this slight, non-significant, change is observed because the negative component of the original signal is influenced by initial OPs overlapping the a-wave.

The amplitudes of OP2 (Fig. 6a) and OP4 (Fig. 6c) decreased with increasing cutoff frequency of the filter. The $50 \%$ threshold was $81.4 \pm 6.1 \mathrm{~Hz}$ and $75.5 \pm 5.1 \mathrm{~Hz}$, respectively, while the amplitude of OP3 increased with increasing cutoff frequency of the filter with the $50 \%$ threshold at $75.4 \pm 6.1 \mathrm{~Hz}$. OP2 and OP4 showed higher amplitudes at low cutoff frequencies and lower amplitudes and high cutoff frequencies with significant differences even after applying multiple comparison's correction $(p<0.001)$ to the pairwise comparisons (OP2: $60-65 \mathrm{~Hz}$ vs. $95-100 \mathrm{~Hz}, 70-75 \mathrm{~Hz}$ vs. $90-105 \mathrm{~Hz}$, $80 \mathrm{~Hz}$ vs. $105 \mathrm{~Hz}$; OP4: $65-70 \mathrm{~Hz}$ vs. $75-105 \mathrm{~Hz}$ ). Calculating the frequency limits of all transition zones, a range between 68.9 and $83.9 \mathrm{~Hz}$ with a frequency of $75.9 \pm 3.5 \mathrm{~Hz}$ at $50 \%$ threshold was found. Peak times of the fast components were relatively constant among the 10 fixed cutoff filters and the variable filter (OP2: $p>0.52$; OP $3: p>0.03$; OP4: $p>0.49$ ), as shown in Fig. 6d-e.

\section{Discussion}

Dark-adapted (DA; scotopic) oscillatory potentials (OPs) showed comparable amplitudes and peak times when they were extracted from the original ERG signals using post hoc filtering at frequencies between 60 and $105 \mathrm{~Hz}$. Moreover, the amplitudes of isolated DA a-wave and b-wave were relatively stable when OPs were removed from the original signals using cutoff frequencies between 75 and $105 \mathrm{~Hz}$ (Fig. 7a). In addition, DA low-frequency components (a-wave and b-wave) obtained with the variable filter resembled those obtained with cutoff filters at this frequency interval (75 to $105 \mathrm{~Hz}$ ) and the original signal. In contrast, significant changes were found in lightadapted (LA; photopic) ERG, b-wave (Fig. 5b) and OP (Fig. 6a-c) amplitudes depending on the filter cutoff frequency. A transition of the b-wave amplitude was observed from relatively stable low values to high values in a frequency range with 50\% threshold at 79.4 $( \pm 4.5) \mathrm{Hz}$, in average. LA b-wave was affected by all cutoff frequencies, as expected from a previous report [14], because of OP4 and b-wave coincident peak 

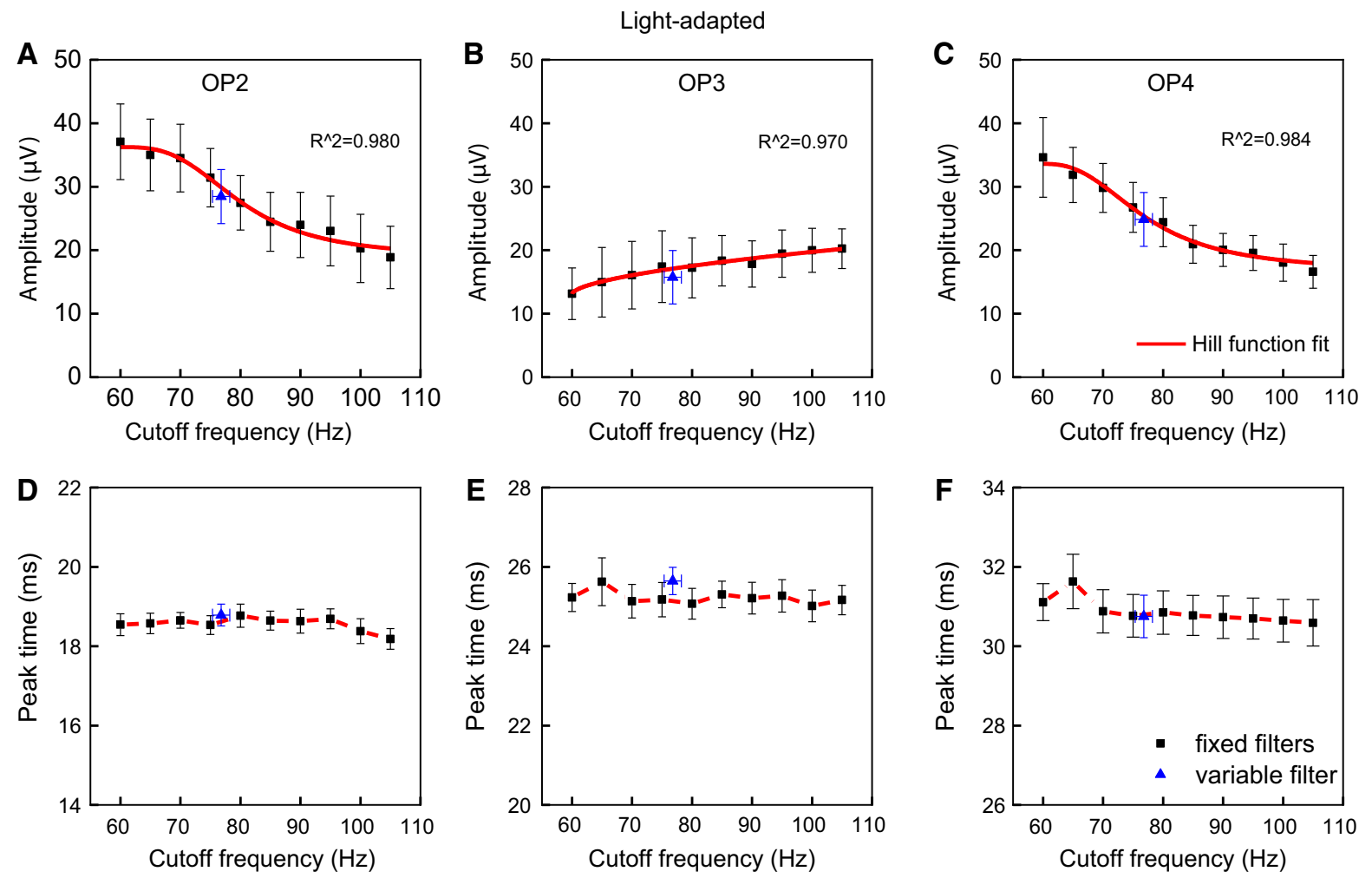

Fig. 6 Light-adapted (LA) fast components (OP2, OP3, and $\mathrm{OP} 4)$ as a function of cutoff frequencies. Mean ( \pm one standard error) amplitudes was fitted with the Hill function (upper row) and peak times (lower row) at each cutoff frequency set for the fixed filters (connected black symbols) and average ( \pm standard

times. In addition, the application of the variable filter based on the minimum amplitude of the LA temporal frequency spectrum (see Fig. 2) resulted in amplitudes and peak times similar to the ones obtained with $50 \%$ threshold of the fitted Hill function at $79.4( \pm 4.5) \mathrm{Hz}$. In summary, the application of the variable filter that effectively separates low- and high-frequency components in mice $[13,15,16]$ does not seem to be necessary for analyzing human DA and LA ERGs. We recognize that flash strength of $3 \mathrm{~cd} . \mathrm{s} / \mathrm{m}^{2}$ does not provide well-defined LA OPs [17], however this is the ISCEV standard flash strength widely used in clinical examinations.

LA OP2 and OP4 amplitudes decreased as the cutoff frequency increased while LA OP3 amplitudes increased with increasing the cutoff frequency. Therefore, high-pass filters with cutoff frequencies set at lower values (separating high and low frequency components below $68.9 \mathrm{~Hz}$ according to present data) error) variable filter (isolated blue symbols) are shown. A transition range of the fast components is between 68.9 and $83.9 \mathrm{~Hz}$ with a frequency of $75.9( \pm 3.5) \mathrm{Hz}$ at $50 \%$ threshold of the Hill function

may emphasize OP2 and OP4, while in the lower frequency range it may generate a more isolated b-wave, less contaminated by the OP frequencies. In contrast, higher cutoff frequencies $(90-105 \mathrm{~Hz})$ beyond the transition range, result in larger $b$-wave and lower OP amplitudes, most likely due to b-waveOP4 interactions (coinciding peaks). The present results, therefore, confirm previous investigations showing that specific cutoff frequencies may influence the main ERG components, particularly the photopic b-wave (Fig. 7b), as well as the extraction of photopic OPs [18]. These findings highlight the need for further clinical investigations concerning the influence of filtering frequencies to isolate slow and fast components of the ERG in different retinal conditions.

OPs are elicited at the same time or very closely synchronized with the slow ERG components [19, 20]. Frequency spectrum analysis and filtering methods to derive human OPs from the original ERG signals have 
Fig. 7 Slow components (awave and b-wave) derived from the original ERG signals (grey traces) using 10 fixed cutoff frequencies (colored traces). Amplified plots for the peak responses are shown. Isolated DA a-wave and b-wave are relatively stable when OPs are excluded from the original signals and they resemble those obtained with the original signal (a). LA a-wave and b-wave changed significantly with OPs excluded from the signal (b). The changes depended on the cutoff frequency: as higher the cutoff frequency higher the b-wave amplitude and peak time (more delayed response)
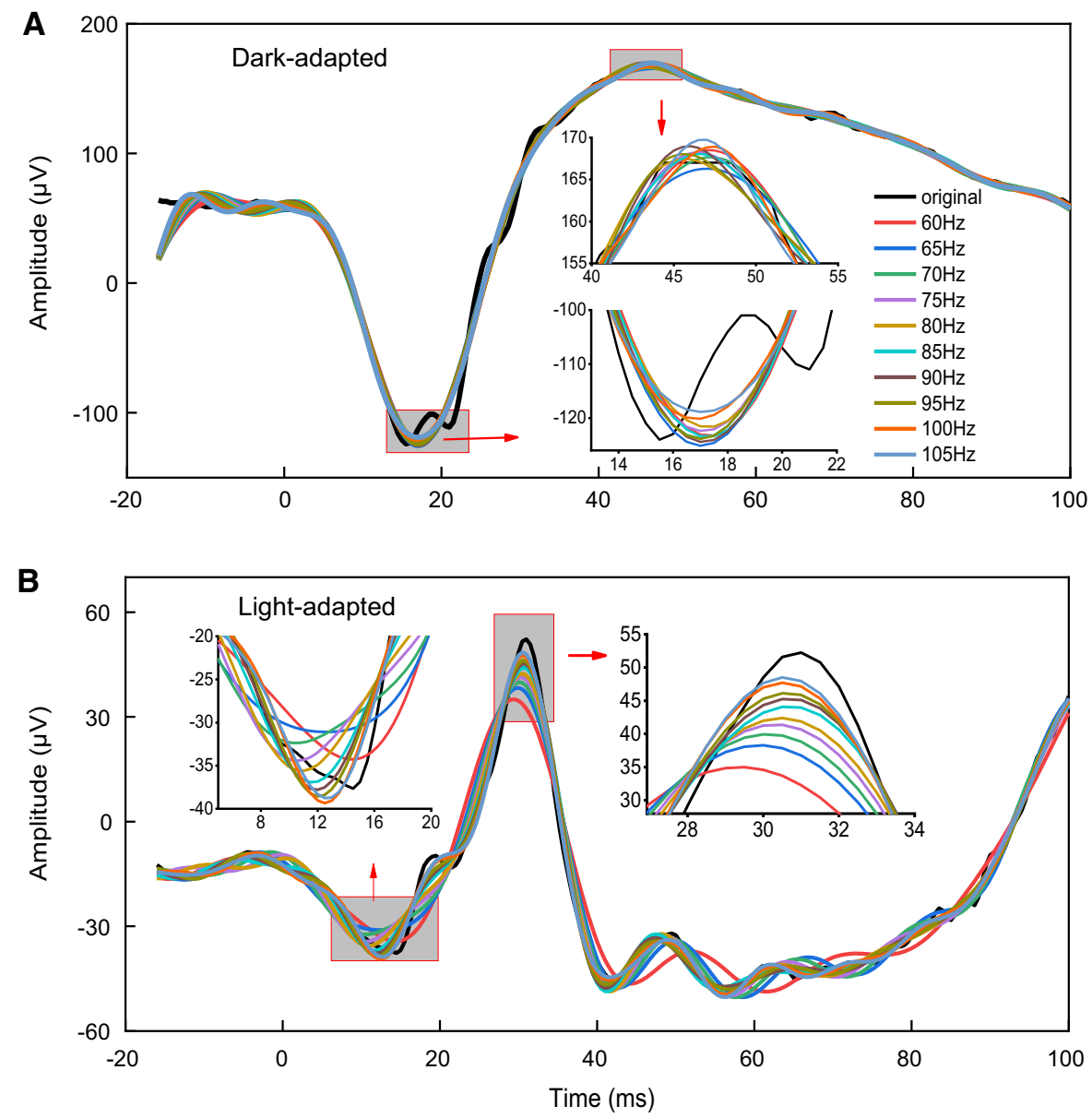

been extensively investigated in the past decades $[3-5,10,12,21-25]$. It has been reported that the main components of the DA ERG influence OP amplitudes, as the slow ERG components (a-wave and b-wave), usually measured with a broad-spectrum band up to $300 \mathrm{~Hz}$, can also be contaminated with high frequency components. Following ISCEV standard recommendations [11], digital filters are usually integrated to the software in commercially available ERG systems with fixed cutoff frequencies, generally between 75 and $100 \mathrm{~Hz}$, to record isolated OPs in the DA ERG protocol. The present results show that indeed at this frequency range DA OP amplitudes and peak times are nearly indistinguishable independent of the specific frequency used. Moreover, if OPs are removed from the original DA signal allowing the isolated analysis of slow components (a-wave and b-wave) using cutoff frequencies between 75 and $100 \mathrm{~Hz}$, the original amplitudes and peak times of the a-wave and the b-wave may only change slightly (Fig. 7a). Therefore, cutoff frequencies between 75 and $100 \mathrm{~Hz}$, as recommended by ISCEV, may be used to derive DA OPs from ERG signals as long as the same filter is used in a single clinical or research center allowing reliable comparisons among subjects. The present results showed that in humans the application of the variable filter provides results similar to the ones obtained with the original signal (without excluding the OPs) for the main ERG components (a-wave and b-wave). Moreover, DA OP amplitudes and peak times using the variable filter $($ mean $=78.2 \mathrm{~Hz})$ resembled those obtained with the ISCEV standard $75 \mathrm{~Hz}$ filter.

LA OPs differ from DA OPs in terms of amplitude, peak time, and frequency, since LA ERG spectrum has two main bands of high temporal frequencies, one between 70 and $80 \mathrm{~Hz}$ and the other between 130 and $160 \mathrm{~Hz}$ [9, 26-28]. The characterization of LA OPs with bandpass filtering has been recently reported by 
Gauthier et al. [12]. Previously, the same group reported a series of experiments using different types of bandpass filters combined with variable bandwidth ranges and, in addition to FFT, continuous wavelet transform (CWT), and discrete wavelet transform (DWT) were applied for a detailed characterization of the LA signals. In summary, Gauvin.et al.'s investigations demonstrated that the frequency distribution of the LA OPs is bimodal and spectral components manipulation may considerably influence LA ERG responses in pathological conditions [27-29]. As isolated LA OPs are usually not considered in the clinical context they may be useful in identifying early pathological changes in the retina [28, 30].

In the LA condition, slow (a-wave and b-wave) and fast (OPs) human ERG components are dependent on each other supporting the view that LA b-wave is shaped by the OPs. This, in turn, is supported by the findings that LA OP (summed) amplitude is proportional to the b-wave amplitude in normal eyes [18]. It has been long proposed that slow and fast ERG components do not share the same cellular origin since a-wave and b-wave mainly reflect photoreceptoral and bipolar cell activation, respectively [31, 32], while the generation of the OPs is attributed to inner retinal structures upstream the ganglion cells [33], most likely generated by neural feedbacks responsible for inhibitory synaptic transmissions [34, 35]. Accordingly, altered b-waves with normal OPs and vice-versa are often reported. For instance, slightly reduced to completely abolished OPs without a-wave and / or b-wave alterations have been found before any detectable clinical sign of diabetic retinopathy (DR). Moreover, LA OP changes may predict the progression of DR [36-38] and can be useful to evaluate toxic effects of pharmacological treatments and the subsequent retinal recovery following treatment discontinuation [39, 40]. Although amacrine cells are generally well-accepted as the OP generators [41, 42] with possible contribution from the bipolar cells [43], OPs may be sensitive to microvascular alterations of the retina [5]. The specific retinal origins of the OPs is also supported by the findings that LA OP2 and OP3 are absent in congenital stationary night blindness, while OP4 is spared [44]. Individual OPs may indeed be generated by specific structures of the retina [42], since pharmacological interventions blocking the activation of specific cells affect differently the OPs [45]. A selective origin of individual OPs emphasizes their clinical value [46], in addition to the clinical relevance of the main ERG components. The present data show that LA b-wave and OPs are differently affected by the cutoff frequency of the digital filters applied to original ERG signals in healthy retinas. Moreover, we found that cutoff frequencies above $100 \mathrm{~Hz}$ would result in LA a- and b-wave amplitudes remarkably similar to the amplitudes of the original signal, meaning that at this frequency the electrophysiologist could be safe to isolate LA OPs that should not be contaminated by the components of the b-wave. However, one needs to consider that the filtered frequencies (i.e.: below $100 \mathrm{~Hz}$ ) also influencing OP amplitudes (mainly LA OP2 and OP4), are eliminated.

The study aimed to describe the effects of cutoff frequencies and the variable filter on low-frequency and high-frequency components. Further investigations of LA ERG signals using more complex timefrequency analysis and evaluating conditions affecting specific retinal structures may provide additional information regarding the application of specific cutoff frequencies in human LA ERGs.

\section{Conclusion}

The present study investigated the effects of fixed cutoff frequency filtering on dark-adapted and lightadapted ERG components and the application of a variable filter showing that LA b-wave and OPs may have different amplitudes and peak times depending on the cutoff frequency in healthy ERG signals. Data obtained with the variable filter are comparable to those obtained with fixed filters. Further investigations may consider applying this analysis to ERG signals recorded from eyes with retinal diseases affecting the inner retina such as those specifically affecting LA OPs. It may also be worthy to investigate it in retinal conditions affecting photoreceptors and / or bipolar cells to evaluate the isolated (OP-free) LA b-wave.

Acknowledgements We thank the National Research, Development, and Innovation Fund of Hungary OTKA (PD134799) to MTSB, Tempus Public Foundation in Hungary (Grant Number 465858) to MTSB and the János Bolyai Scholarship of the Hungarian Academy of Sciences to BVN. We also thank Sao Paulo Research Foundation (FAPESP 2014/26818-2) and CNPq 1A productivity fellowship to DFV. The research reported in this paper and carried out at BME has been supported by the NRDI Fund (TKP2020 IES, Grant No. BME-IE-BIO) based on the charter of bolster issued by the 
NRDI Office under the auspices of the Ministry for Innovation and Technology. The research reported in this paper and carried out at BME has been supported by the NRDI Fund (TKP2020 NC, Grant No. BME-NC) based on the charter of bolster issued by the NRDI Office under the auspices of the Ministry for Innovation and Technology.

Funding Open access funding provided by Budapest University of Technology and Economics. National Research, Development, and Innovation Fund of Hungary OTKA (PD134799) and Tempus Public Foundation (Grant Number 465858) to MTSB, Sao Paulo Research Foundation (FAPESP Grant Number 2014/26818-2 to DFV). CNPq 1A productivity fellowship to DFV. Author BVN was supported by the János Bolyai Scholarship of the Hungarian Academy of Sciences.

\section{Declarations}

Conflict of interest All authors certify that they have no affiliations with or involvement in any organization or entity with any financial interest (such as honoraria; educational grants; participation in speakers' bureaus; membership, employment, consultancies, stock ownership, or other equity interest; and expert testimony or patent-licensing arrangements), or non-financial interest (such as personal or professional relationships, affiliations, knowledge or beliefs) in the subject matter or materials discussed in this manuscript.

Ethical approval All procedures performed in studies involving human participants were in accordance with the ethical standards of the Institutional ethics committee of the University of Sao Paulo in Brazil (CEP-HU/USP 156.826) and with the 1964 Helsinki declaration and its later amendments or comparable ethical standards. Informed consent was obtained from all individual participants included in the study.

Open Access This article is licensed under a Creative Commons Attribution 4.0 International License, which permits use, sharing, adaptation, distribution and reproduction in any medium or format, as long as you give appropriate credit to the original author(s) and the source, provide a link to the Creative Commons licence, and indicate if changes were made. The images or other third party material in this article are included in the article's Creative Commons licence, unless indicated otherwise in a credit line to the material. If material is not included in the article's Creative Commons licence and your intended use is not permitted by statutory regulation or exceeds the permitted use, you will need to obtain permission directly from the copyright holder. To view a copy of this licence, visit http://creativecommons.org/licenses/by/4.0/.

\section{References}

1. Algvere P (1968) Clinical studies on the oscillatory potentials of the human electroretinogram with special reference to the scotopic b-wave. Acta Ophthalmol (Copenh) 46:993-1024
2. Cobb WA, Morton HB (1953) A new component of the human electroretinogram. J Physiol 123:30

3. Gur M, Zeevi Y (1980) Frequency-domain analysis of the human electroretinogram. J Opt Soc Am 70:53-59. https:// doi.org/10.1364/josa.70.000053

4. McCulloch C, Orpin JA, Waisberg JW, Parker JA (1972) Frequency analysis of the human dark adapted electroretinogram. Can J Ophthalmol 7:189-198

5. Speros P, Price J (1981) Oscillatory potentials. History, techniques and potential use in the evaluation of disturbances of retinal circulation. Surv Ophthalmol 25:237-252. https://doi.org/10.1016/0039-6257(81)90093-x

6. Wachtmeister L (1998) Oscillatory potentials in the retina: what do they reveal. Prog Retin Eye Res 17:485-521. https://doi.org/10.1016/s1350-9462(98)00006-8

7. Yin J, Lei B, Peng H et al (2011) Characteristics of darkadapted and light-adapted oscillatory potentials in human electroretinogram. J South Med Univ 31:2057-2060

8. Bui BV, Armitage JA, Vingrys AJ (2002) Extraction and modelling of oscillatory potentials. Doc Ophthalmol 104:17-36. https://doi.org/10.1023/A:1014401502915

9. Dimopoulos IS, Freund PR, Redel T et al (2014) Changes in rod and cone-driven oscillatory potentials in the aging human retina. Invest Ophthalmol Vis Sci 55:5058-5073. https://doi.org/10.1167/iovs.14-14219

10. Severns ML, Johnson MA, Bresnick GH (1994) Methodologic dependence of electroretinogram oscillatory potential amplitudes. Doc Ophthalmol 86:23-31. https://doi.org/ 10.1007/BF01224625

11. McCulloch DL, Marmor MF, Brigell MG et al (2015) ISCEV Standard for full-field clinical electroretinography (2015 update). Doc Ophthalmol 130:1-12. https://doi.org/ 10.1007/s10633-014-9473-7

12. Gauthier M, Gauvin M, Lina J-M, Lachapelle P (2019) The effects of bandpass filtering on the oscillatory potentials of the electroretinogram. Doc Ophthalmol 138:247-254. https://doi.org/10.1007/s10633-019-09683-w

13. Harazny J, Scholz M, Buder T et al (2009) Electrophysiological deficits in the retina of the DBA/2J mouse. Doc Ophthalmol 119:181-197. https://doi.org/10.1007/s10633009-9194-5

14. Peachey NS, Alexander KR, Derlacki DJ et al (1991) Effects of light adaptation on the response characteristics of human oscillatory potentials. Electroencephalogr Clin Neurophysiol 78:27-34. https://doi.org/10.1016/00134694(91)90015-v

15. Barboni MTS, Vaillend C, Joachimsthaler A et al (2020) Rescue of defective electroretinographic responses in Dp71-null mice with AAV-mediated reexpression of Dp71. Invest Ophthalmol Vis Sci 61:11. https://doi.org/10.1167/ iovs.61.2.11

16. Barboni MTS, Liber AMP, Joachimsthaler A et al (2021) Altered visual processing in the mdx52 mouse model of Duchenne muscular dystrophy. Neurobiol Dis. https://doi. org/10.1016/j.nbd.2021.105288

17. Rufiange M, Rousseau S, Dembinska O, Lachapelle P (2002) Cone-dominated ERG luminance-response function: the Photopic Hill revisited. Doc Ophthalmol 104:231-248. https://doi.org/10.1023/a:1015265812018

18. Lachapelle P (1990) Oscillatory potentials as predictors to amplitude and peak time of the photopic b-wave of the 
human electroretinogram. Doc Ophthalmol 75:73-82. https://doi.org/10.1007/BF00142596

19. Brunette JR (1972) Double a-waves and their relationships to the oscillatory potentials. Invest Ophthalmol 11:199-210

20. Brunette JR, Desrochers R (1970) Oscillatory potentials: a clinical study in diabetics. Can J Ophthalmol 5:373-380

21. Algvere P, Westbeck S (1972) Human ERG in response to double flashes of light during the course of dark adaptation: a Fourier analysis of the oscillatory potentials. Vision Res 12:195-214. https://doi.org/10.1016/0042-6989(72)901113

22. Poppele RE, Maffei L (1967) Frequency analysis of the electroretinogram. J Neurophysiol 30:982-992. https://doi. org/10.1152/jn.1967.30.5.982

23. Vallabhan G, Kristiansen S, Price J, Young RS (1988) Effect of adaptation and wavelength on the power spectrum of human oscillatory potentials. Doc Ophthalmol 69:145-151. https://doi.org/10.1007/bf00153695

24. Van der Torren K, Groeneweg G, van Lith G (1988) Measuring oscillatory potentials: fourier analysis. Doc Ophthalmol 69:153-159. https://doi.org/10.1007/bf00153696

25. Viet Bui B, Andrew Armitage J, Jonas Vingrys A (2002) Extraction and modelling of oscillatory potentials. Doc Ophthalmol 104:17-36

26. Derr PH, Meyer AU, Haupt EJ, Brigell MG (2002) Extraction and modeling of the oscillatory potential: signal conditioning to obtain minimally corrupted oscillatory potentials. Doc Ophthalmol 104:37-55. https://doi.org/10. 1023/a:1014474026114

27. Gauvin M, Lina J-M, Lachapelle P (2014) Advance in ERG analysis: from peak time and amplitude to frequency, power, and energy mathieu. Hindawi Publ Corp. https://doi. org/10.1155/2014/246096

28. Gauvin M, Dorfman AL, Trang N et al (2016) Assessing the contribution of the oscillatory potentials to the genesis of the photopic ERG with the discrete wavelet transform. Hindawi Publ Corp. https://doi.org/10.1155/2016/2790194

29. Gauvin M, Little JM, Lina J-M, Lachapelle P (2015) Functional decomposition of the human ERG based on the discrete wavelet transform. J Vis 15:14. https://doi.org/10. 1167/15.16.14

30. Holopigian K, Seiple W, Lorenzo M, Carr R (1992) A comparison of photopic and scotopic electroretinographic changes in early diabetic retinopathy. Invest Ophthalmol Vis Sci 33:2773-2780

31. Frishman LJ (2006) Origins of the electroretinogram. Principles and practice of clinical electrophysiology of vision, 2nd edn. MIT Press, Cambridge, pp 139-183

32. Robson JG, Saszik SM, Ahmed J, Frishman LJ (2003) Rod and cone contributions to the a-wave of the electroretinogram of the macaque. J Physiol (Lond) 547:509-530. https://doi.org/10.1113/jphysiol.2002.030304

33. Doty RW, Kimura DS (1963) Oscillatory potentials in the visual system of cats and monkeys. J Physiol (Lond)
168:205-218. sp007187

https://doi.org/10.1113/jphysiol.1963.

34. Brown KT (1968) The eclectroretinogram: its components and their origins. Vision Res 8:633-677. https://doi.org/10. 1016/0042-6989(68)90041-2

35. Heynen H, Wachtmeister L, van Norren D (1985) Origin of the oscillatory potentials in the primate retina. Vision Res 25:1365-1373. 6989(85)90214-7

36. Bresnick GH, Korth K, Groo A, Palta M (1984) Electroretinographic oscillatory potentials predict progression of diabetic retinopathy. Prelim Rep Arch Ophthalmol 102:1307-1311. https://doi.org/10.1001/archopht.1984. 01040031057023

37. Tzekov R, Arden GB (1999) The electroretinogram in diabetic retinopathy. Surv Ophthalmol 44:53-60. https://doi. org/10.1016/s0039-6257(99)00063-6

38. Yonemura D, Aoki T, Tsuzuki K (1962) Electroretinogram in diabetic retinopathy. Arch Ophthalmol 68:19-24. https:// doi.org/10.1001/archopht.1962.00960030023005

39. Morong S, Westall CA, Nobile R et al (2003) Longitudinal changes in photopic OPs occurring with vigabatrin treatment. Doc Ophthalmol 107:289-297. https://doi.org/10. 1023/b:doop.0000005338.51554.e3

40. Westall CA, Nobile R, Morong S et al (2003) Changes in the electroretinogram resulting from discontinuation of vigabatrin in children. Doc Ophthalmol 107:299-309. https:// doi.org/10.1023/b:doop.0000005339.23258.8f

41. Ogden TE (1973) The oscillatory waves of the primate electroretinogram. Vision Res 13:1059-1074. https://doi. org/10.1016/0042-6989(73)90144-2

42. Wachtmeister L, Dowling JE (1978) The oscillatory potentials of the mudpuppy retina. Invest Ophthalmol Vis Sci 17:1176-1188

43. Wachtmeister L (1987) Basic research and clinical aspects of the oscillatory potentials of the electroretinogram. Doc Ophthalmol 66:187-194. https://doi.org/10.1007/ bf00145232

44. Lachapelle P, Little JM, Polomeno RC (1983) The photopic electroretinogram in congenital stationary night blindness with myopia. Invest Ophthalmol Vis Sci 24:442-450

45. Gutiérrez O, Spiguel RD (1973) Oscillatory potentials of the cat retina: effects of adrenergic drugs. Life Sci 13:991-999. https://doi.org/10.1016/0024-3205(73)90090-8

46. Yonemura D, Kawasaki K (1979) New approaches to ophthalmic electrodiagnosis by retinal oscillatory potential, drug-induced responses from retinal pigment epithelium and cone potential. Doc Ophthalmol 48:163-222. https:// doi.org/10.1007/BF00207350

Publisher's Note Springer Nature remains neutral with regard to jurisdictional claims in published maps and institutional affiliations. 